\title{
Why can't planets be like stars?
}

\section{Planetary science: both the deductive skills of geologists and the mathematical approach of astrophysicists are needed to study planets.}

\section{Stua} tuart Ross Taylor System we have eight planets, all of which are distinct from one another in mass, density, composition, rotation rates and angle of inclination (obliquity). Their only common properties are nearcircular orbits and low inclinations to the Earth-Sun plane, characteristics that enabled Pierre-Simon Laplace to conclude in 1796 that they had originated from a rotating disk, the solar nebula.

Our planetary system also includes more than 120 moons and a host of smaller bodies, most of which exhibit some peculiarities of composition or behaviour. Thus, there appears to be no uniformity in the processes of planetary or satellite formation from the gases, ices and rocky components of the primordial nebula. Planets may also migrate from their original positions, effectively randomizing any initial radial variations in the chemistry of the nebula.

The discovery of more than 100 planets orbiting stars other than the Sun has brought the question of planetary origin and evolution into sharp focus. Our limited sampling of these extrasolar planets reveals even wider variations in terms of mass and spacing of planets and - to add additional complexity - many of these newly discovered planets are in highly elliptical orbits. It seems likely that we may eventually find that planets forming from disks rotating around young stars will occupy all available niches within the limits imposed by the cosmochemical abundances of the elements and the laws of physics and chemistry.

Unlike planets, stars are relatively uniform in composition and differ mostly in mass. The basic features of stellar evolution have long been understood and are described by the Hertzsprung-Russell diagram, which relates surface temperature to luminosity and essentially reflects the nuclear reactions taking place in stars.

By contrast, planets are individuals that show few systematic relationships and have resisted attempts at classification or even definition, as witnessed in the furore over the status of Pluto, which is an eccentric dwarf when placed among the planets, but is better suited to be the king of the many icy bodies in the Kuiper belt. So far, there is no planetary equivalent of the Hertzsprung-Russell diagram. Even if we arrive at a satisfactory explanation for the formation and evolution of our planetary system, there is no guarantee that this will apply elsewhere. Perhaps this is the reason, as Stephen Brush has commented, that the origin of the Solar System represents one of the oldest unsolved problems in science.

The problems of studying planets are well illustrated by the history of attempts to understand the Earth. Geology was a latecomer among the sciences. Even after James Hutton's insights into deep time in 1788, it was a further 150 years before plate tectonics was understood as the mechanism responsible for the architecture of the Earth's surface. Plate tectonics has the useful property of both building continents and forming ore deposits useful for advanced civilizations in doing so, enabling this discussion to take place. But this process is unique to the Earth among the planets of the Solar System and was only made possible by the late stochastic addition of a water content of a few hundred parts per million. Many of the difficulties in trying to understand the evolution of the Moon arose from the uncritical attempts to apply our hard-won experience with wetter terrestrial rocks to those from our bone-dry satellite.

Even when nature got around to building two similar planets, it finished up with the Earth and Venus. These twins are close in mass, density, bulk composition and the abundances of the heat-producing elements (potassium, uranium and thorium).
But Venus is a one-plate planet without a moon and seems to undergo planetary-wide resurfacing with basalt perhaps once every billion years. What causes the difference between the geological histories of these twins? The short answer is water, but much may be due to variations in the early history of impacts during planetary accretion. Similarity is not identity, and the Earth resembles Venus much as $\mathrm{Dr}$ Jekyll resembled Mr Hyde. As we search for terrestrial-like planets elsewhere, we need to find out the reasons for these differences and the conditions that allow these diverse bodies to form at all.

The study of planets represents a new area in scientific nquiry, just as geology did in the nineteenth century. While one might intuitively think that the rocky planets could be left to the geologists and the gas giants to the astronomers, neither group alone seems well suited to producing the necessary synthesis. Geologists deal mostly with surface observations, but planetary crusts differ greatly from interiors. In contrast, astronomers have long been involved with the internal evolution of stars. No single specialist group seems well equipped to handle the diversity of planetary systems and the philosophical problems in dealing with stars, the Earth and the variety of solar and extrasolar planets. A unifying approach is needed to avoid the dilemma of the six wise but blind men confronted with an elephant. Just as astrophysics, geochemistry, biochemistry and geophysics have risen at the boundaries between the classical sciences, so planetary science now requires new types of investigators. Such investigators need a distinct mindset somewhere between the approaches of astronomers - who want to treat planets mathematically like stars - and geologists, who want to generalize from their detective-like experience with the Earth.

Stuart Ross Taylor is in the Department of Earth and Marine Sciences, Australian National

University, Canberra ACT 0200, Australia.

\section{FURTHER READING}

Brush, S. G. A History of Modern Planetary Physics Vol. 3 (Cambridge Univ. Press, 1996).

Stevenson, D. J. Phys. Today 57, 43-48 (2004). Taylor, S. R. Destiny or Chance: Our Solar System and its Place in the Cosmos (Cambridge Univ. Press, 2000). 